University of Nebraska - Lincoln

DigitalCommons@University of Nebraska - Lincoln

\title{
Guided self-assembly of diblock copolymer thin films on chemically patterned substrates
}

\author{
Xiang-Fa Wu \\ University of Nebraska - Lincoln \\ Yuris A. Dzenis \\ University of Nebraska-Lincoln, ydzenis@unl.edu
}

Follow this and additional works at: https://digitalcommons.unl.edu/cmrafacpub

Part of the Nanoscience and Nanotechnology Commons

Wu, Xiang-Fa and Dzenis, Yuris A., "Guided self-assembly of diblock copolymer thin films on chemically patterned substrates" (2007). Faculty Publications from Nebraska Center for Materials and Nanoscience. 18.

https://digitalcommons.unl.edu/cmrafacpub/18

This Article is brought to you for free and open access by the Materials and Nanoscience, Nebraska Center for (NCMN) at DigitalCommons@University of Nebraska - Lincoln. It has been accepted for inclusion in Faculty Publications from Nebraska Center for Materials and Nanoscience by an authorized administrator of DigitalCommons@University of Nebraska - Lincoln. 


\title{
Guided self-assembly of diblock copolymer thin films on chemically patterned substrates
}

\author{
Xiang-Fa $\mathrm{Wu}^{\mathrm{a})}$ and Yuris A. Dzenis \\ Department of Engineering Mechanics, Center for Materials Research and Analysis, \\ University of Nebraska-Lincoln, Lincoln, Nebraska 68588-0526
}

(Received 3 January 2006; accepted 21 September 2006; published online 3 November 2006)

\begin{abstract}
We study the guided self-assembly of symmetric/asymmetric diblock copolymer (BCP) films on heterogeneous substrates with chemically patterned surface by using a coarse-grained phase-separation model. During the procedure, the free energy employed for the BCP films was modeled by the Ginzburg-Landau free energy with nonlocal interaction, and the flat, chemically patterned surface was considered as a heterogeneous surface with short-range interaction with the BCP molecules. The resulting Cahn-Hilliard equation was solved by means of an efficient semi-implicit Fourier-spectral algorithm. Effects of pattern scale, surface chemical potential, and BCP asymmetry on the self-assembly process were explored in detail and compared with those without chemically patterned substrate surfaces. It was found that the morphology of both symmetric and asymmetric BCP films is strongly influenced by the commensurability between the unconstrained natural period $\lambda^{*}$ of the bulk BCP and the artificial pattern period. Simulation shows that patterned surface with period close to $\lambda^{*}$ leads to highly ordered morphology after self-assembly for both symmetric and asymmetric BCP films, and it also dramatically accelerates the guided self-assembly process. The present simulation is in a very good agreement with the recent experimental observation in BCP nanolithography. Finally, the present study also expects an innovative nanomanufacturing method to produce highly ordered nanodots based on the guided self-assembly of asymmetric BCP films on chemically patterned substrates. (0) 2006 American Institute of Physics. [DOI: 10.1063/1.2363982]
\end{abstract}

\section{INTRODUCTION}

Diblock copolymer (BCP) molecules are made of two thermodynamically incompatible polymeric chains through covalent bonds. ${ }^{1-3}$ Due to their unique chain structures and chemophysical properties, BCPs usually form a wide variety of fascinating microstructures in bulk and in solution in the form of spheres, cylinders, bicontinuous, perforated layers, or lamellae through molecular self-assembly under proper conditions such as annealing. ${ }^{1-3}$ These microstructures have the typical length scale comparable to the size of the BCP molecules (typically $5-50 \mathrm{~nm}$ ). Under proper external fields, the BCP molecular chains can be guided to generate various patterns in nanoscale. This is one of the most attractive to materials scientists and engineers in the field of nanomaterial synthesis and nanodevice fabrication through molecular self-assembly. ${ }^{4-11}$ In the past few years the guided selfassembly of BCP films on patterned substrates has evoked considerable interest due to their controllable microstructures in nanoscale and potential applications in nanotechnologies ${ }^{12-21}$ such as high-density random-access memory devices, increased capacitance gate devices, acoustic transducer arrays, photonic imaging arrays, and biomedical implant modifications, etc. In order to control and optimize the nanomanufacturing process and to produce targeted

\footnotetext{
${ }^{\text {a) }}$ Author to whom correspondence should be addressed. Fax: 402-472-8292 Electronic mail: xfwu@unlserve.unl.edu
}

final nanoproducts, it is desirable to understand and therefore simulate this unique molecular self-assembly process in the vicinity of substrates.

In principle, molecular self-assembly in BCPs is described in terms of phase separation that takes place in $\mathrm{BCP}$ melts or blends after a quench into the unstable region of the phase diagram. So far, phase-separation behavior in bulk BCPs has been studied extensively in literature based largely on cell dynamics simulation, where periodic boundary conditions were usually employed. As a matter of fact, surface and BCP dimensions in bulk BCPs do not affect the simulation results. However, for an ultrathin copolymer film, the spatial isotropy/symmetry of the system is broken due to the surface effect. As a result, surface/substrate plays a vital role in the molecular self-assembly process in a thin BCP film. Surface/substrate can be further patterned either chemically or physically to govern the phase-separation process for specific purpose in practice. Theoretically, within the framework of self-consistent field theory, ${ }^{22-26}$ Petera and Muthukumar $^{27,28}$ investigated the density profiles of thin symmetric BCP films confined on flat, chemically patterned surfaces. Through asymptotic analysis, they obtained the near-surface density profile in the cross section of the BCP films above and below the order-disorder transition (ODT). They examined the effect of the ratio of the pattern period to the radius of gyration of the BCP molecular chains, Flory parameter that measures the interaction energy between two monomers, and the surface interaction on the density profile 
in detail. For a patterned surface with pattern period greater than the natural period of a symmetric BCP in bulk, the lamellar ordering in the BCP film always tries to accommodate the surface pattern length scale by tilting the orientation of the lamellae with respect to the surface according to an angle $\theta$ given by $\sin \theta=L_{0} / L$, where $L_{0}$ is the natural period of the BCP in bulk and $L$ is the artificial pattern period. When $L_{0}=L$, the lamellae are perpendicular to the patterned surfaces. For the surface pattern period smaller than the natural period of the BCP in bulk, the diblocks close to the patterned surfaces are compressed and twisted in order to accommodate the surface pattern period and then shortly relaxed away from the surfaces. By using largely the same method, Tsori and Andelman ${ }^{29-31}$ further considered the surface free energy with a quadratic relation with respect to the monomer density close to the substrate surfaces. Their simulations confirmed Petera and Muthukumar's conclusion in a broad sense. Furthermore, by means of Fourier analysis, Tsori and Andelman obtained the in-planar density profile of symmetric BCP films confined on patterned surfaces. Due to the above analyses based on the asymptotic analysis of the free energy in equilibrium, they were unable to predict the density profile evolution and to estimate the effect of random fluctuation on the monomer density profile, etc. Therefore, more detailed simulation is still desired.

On the other hand, numerical simulation based on coarse-grained phase-separation modeling ${ }^{32-38}$ and lattice Monte Carlo (MC) modeling ${ }^{39-42}$ have been well developed to the understanding of the molecular self-assembly in BCP films confined on flat, chemically patterned surfaces after a quench. Using a three-dimensional (3D) coarse-grained phase-separation model, Chen and Chakrabarti ${ }^{33}$ studied the entire density profile of thin symmetric BCP films confined on flat surfaces that were patterned with alternating parallel strips of surface potential. They found that the in-planar density profile of the BCP film is strongly influenced by the commensurability between the BCP lamellar size $\lambda^{*}$ in bulk and the artificial pattern period $\lambda$ and also affected by the distance from the substrate surface simultaneously. When $\lambda$ $\approx \lambda^{*}$, the ordered morphology of the BCP film has a strong overlap with the preassigned substrate chemical pattern throughout the film corresponding to those observed in recent nanolithographical studies, ${ }^{15,18}$ and it also starts decaying near the free surface for the film thickness over $\lambda^{*}$. However, for $\lambda$ well above and well below $\lambda^{*}$, the overlap strongly depends on the distance from the substrate surface. Ordered lamellae can be found in a specific distance from the substrate surface depending on the strength of the surface potential and the preassigned pattern period. For asymmetric BCP film, using the similar methodology, Podariu and Chakrabarti $^{38}$ investigated the morphology of a thin BCP film after a quench as a function of the composition of the $A / B$ monomers, film thickness, and surface wetting properties. It was found that for a specific monomer composition between two different bulk phases external parameters play a vital role in the morphology transition below ODT. Furthermore, by using a lattice MC model, Semler and Genzer ${ }^{41}$ and Jayaraman et al. ${ }^{42,43}$ considered the recognition of heterogeneous surface using the varying adsorption ability of the
BCP monomers on the substrate surface. It was found that the surface molecular recognition strongly depends on the strength of attraction between the adsorbing surface domains and the copolymer adsorbing segments. A threshold of the attraction strength was identified numerically in their simulation. Beyond this value, the extent to which BCPs transfer the chemical pattern from the surface to the bulk is relatively unaffected by the further increase of the attraction strength. Numerical simulation also indicated that the commensurability between the BCP chain length and the pattern size influences the recognition of the surface pattern. Yet, this correlation is not as pronounced as that found through coarsegrained phase-separation modeling. Nevertheless, numerical studies provide rich space to guide the molecular selfassembly of BCP films for the purpose of nanomanufacturing and nanodevice design in reality.

Experimentally, recent investigations ${ }^{11,13-20}$ have indicated that various patterns of alternating composition in ultrathin BCP films can be achieved on patterned substrates, usually silicon wafers, depending on the choice of pattern period of the alternating strips and spaces created by ultraviolet interferometric lithography or other physical/chemical imprinting methods. As an example, for symmetric lamellaforming styrene-block-methyl methacrylate (PS-b-PMMA) used typically in nanolithography, its lamellar period in bulk state is around $48 \mathrm{~nm}$. In such case, highly ordered parallel lamellae with thickness around $60 \mathrm{~nm}$ have been obtained successfully on patterned substrates in the case of the pattern periods of the alternating strips and spaces very close to its lamellar period in bulk $(\sim 48 \mathrm{~nm}) .{ }^{15,18}$ Experimental observations ${ }^{15,18,20}$ also showed that when the pattern periods slightly below the lamellar periods, the formed lamellae were compressed but were for the most part registered and expitaxial to the surface pattern, and dislocations existed and grew during the entire annealing process. Furthermore, in the case of pattern periods slightly greater than that of the BCPs, wavy ribbon structures were formed with period slightly greater than the lamellar period of the BCP in free bulk state. Moreover, in the case of pattern periods much greater than that of the BCP, the substrate pattern had no significant effect on the lamella formation. These results provided rich information for theoretical analysis and relevant numerical simulation and for the improvement of the nanomanufacturing process.

So far, numerical simulation of the phase separation in BCPs has shown that BCP molecules can be orientated on chemically prepatterned substrates with proper pattern periods. Recent simulation results are in a good agreement with those observed in experiments. Thus, numerical modeling has shown the great power in process analysis and optimization in the coming mass nanomanufacturing. Nevertheless, in an attempt to exploit this novel nanotechnology for practical use in the near future, it is still desirable to conduct more detailed investigation on the effect of substrate pattern and heterogeneities, magnitude of the surface chemical potential, and BCP asymmetry, etc., on the molecular self-assembly process of the BCP films. For example, further study is needed to confirm the compressed lamella structure and dislocations found in recent experiments. ${ }^{15,18}$ Moreover, effi- 
cient and robust numerical methods are always attractive for large area simulation for the purpose of realistic application. As one of these efforts, in this work, we present the results from a detailed numerical study of the guided self-assembly in symmetric/asymmetric BCP films confined on chemically patterned surfaces by solving the resulting Cahn-Hilliard (CH) equation numerically. In this study, the free energy of the BCP systems was modeled by the classic GinzburgLandau free energy functional with nonlocal interaction. $^{22-26,33,38,44,45}$ The flat, hierarchical substrate was dealt with as a patterned surface of alternating parallel strips and spaces of chemical potential with short-range interaction with the BCP monomers. Mosaic-patterned substrate was also employed for comparison. Different from using the conventional explicit finite difference methods for most classic cell dynamics simulations, we adopt an efficient semiimplicit Fourier-spectral method to solve the resulting nonlinear $\mathrm{CH}$ equation. ${ }^{46,47}$ Since this numerical scheme is unconditionally stable, the time steps used for integration can be chosen to be much greater than those used in the conventional coarse-grained phase-separation modeling. We examined the effects of pattern scale and heterogeneity, chemical potential, and BCP asymmetry on the self-assembly process. Specifically, through numerical experiments in this study, we find that, by employing special chemically mosaic-patterned substrate, ultrathin asymmetrical BCP films can be guided to create highly ordered nanodots that are expected to be extremely important to the potential use in high-density random-access memory devices, among others. Consequently, evolution mechanisms and potential applications of the guided self-assembly of BCPs are further addressed.

\section{MODEL AND NUMERICAL PROCEDURE}

In view of the Landau-Ginzburg phenomenological model of phase separation, the time-dependent free energy functional of an asymmetric BCP film confined on a chemically patterned surface can be expressed as ${ }^{22-26,33,38,44,45}$

$$
\begin{aligned}
\frac{F[\varphi(\mathbf{r}, t)]}{k_{B} T}= & \int\left\{f[\varphi(\mathbf{r}, t)]+\frac{K}{2}|\nabla \varphi(\mathbf{r}, t)|^{2}\right\} d \mathbf{r} \\
& +\frac{A}{2} \int G\left(\mathbf{r}, \mathbf{r}^{\prime}, t\right)\left[\varphi(\mathbf{r}, t)-\varphi_{0}\right] \\
& \times\left[\varphi\left(\mathbf{r}^{\prime}, t\right)-\varphi_{0}\right] d \mathbf{r} d \mathbf{r}^{\prime} \\
& -\int B(\mathbf{r})[1+\varphi(\mathbf{r}, t)] / 2 d A,
\end{aligned}
$$

where the first two integrals are related to the bulk free energy and the third is the interfacial energy of the short interaction between the $\mathrm{BCP}$ and the substrate surface. $k_{B}$ is the Boltzmann constant; $T$ is the absolute temperature; $K$ is a constant to delineate the phase boundary energy; $\mathbf{r}$ is the local position vector; $f[\varphi(\mathbf{r}, t)]$ is the local coarse-grained free energy density of mixing that takes the Landau form such that $f[\varphi(\mathbf{r}, t)]=-\varphi(\mathbf{r}, t)^{2} / 2+\varphi(\mathbf{r}, t)^{4} / 4 . \varphi(\mathbf{r}, t)$ is the local concentration difference between the two BCP monomers that are assumed as $A$ and $B$ such that $\varphi(\mathbf{r}, t)=2 \phi(\mathbf{r}, t)-1$, where $\phi(\mathbf{r}, t)$ is the local volume fraction of the $A$ monomer in the $A-B$ BCP. $\varphi_{0}$ is the mean value of the order parameter averaging spatially. $A$ is a factor associated with the nonlocal molecular interaction potential, and $B(\mathbf{r})$ stands for the surface chemical potential that can be patterned artificially for specific purpose. $G$ is Green's function for the Laplace equation, i.e., $\nabla^{2} G=-\delta(\mathbf{r}-\mathbf{r})$. For $\varphi_{0}=0$, the above free energy functional recovers that of a symmetric BCP.

In this study, we assume the BCP film being very thin, i.e., monolayer, ${ }^{14}$ which is also an approximate of the $\mathrm{BCP}$ films used in recent nanolithographical study. ${ }^{15,18,20}$ Therefore, the above integrals can be reduced to those with respect to the substrate surface. After a quench from the hightemperature disordered melting state to a temperature below the microphase segregation temperature, stable phases will form through phase segregation in order to reduce the free energy of the BCP system. This process can be modeled by the $\mathrm{CH}$ equation or model $\mathrm{B}$ in the Hohenberg-Halperin notation $^{48}$ such that

$$
\frac{\partial \varphi}{\partial t}=\nabla\left[M \nabla \frac{\delta F(\varphi, t)}{\delta \varphi}\right],
$$

where $M$ is the mobility that is assumed to be constant for convenience. Substitution of (1) into (2) and then rescaling it with $M$ yields the dimensionless $\mathrm{CH}$ equation on the substrate surface,

$$
\frac{\partial \varphi}{\partial t}=\nabla^{2}\left(-\varphi+\varphi^{3}-\nabla^{2} \varphi-\beta\right)-\alpha\left(\varphi-\varphi_{0}\right)
$$

where $\alpha$ is a compound dimensionless phenomenological parameter characterizing the nonlocal $A-B$ potential, the size of molecular length, and the mobility; $\beta$ is a rescaled function with respect to the local position vector on the substrate surface in the case of a chemically patterned substrate. A constant $\beta$ value corresponds to a neutral or homogeneous substrate. Usually, the greater $\alpha$ value corresponds to the greater physiochemical similarity between the $A$ and $B$ monomers in the BCP.

The $\mathrm{CH}$ equation can be solved conveniently by means of explicit finite difference methods within a representative cell under periodic boundary conditions. Here we adopt an efficient semi-implicit Fourier-spectral method ${ }^{46,47}$ to solve Eq. (3) under the same boundary conditions. Due to its unconditional stability, this numerical scheme allows us to use much greater time steps and to keep a very good numerical stability simultaneously, and therefore dramatically accelerates the numerical simulation. To do so, by performing double Fourier transform with respect to the spatial variables $x$ and $y$ at the two sides of Eq. (3), we obtain the $\mathrm{CH}$ equation (3) in the time-Fourier domain,

$$
\frac{\partial \bar{\varphi}}{\partial t}=k^{2}\left(\bar{\varphi}-\overline{\varphi^{3}}-k^{2} \bar{\varphi}+\bar{\beta}\right)-\alpha\left(\bar{\varphi}-\bar{\varphi}_{0}\right),
$$

where $k=\sqrt{k_{x}^{2}+k_{y}^{2}}$ with $k_{x}$ and $k_{y}$ as the variables in the Fourier domain and variables with upper bars denote the double Fourier transforms of the corresponding variables in the physical space. For instance, $\bar{\varphi}(k, t)$ is the double Fourier transform of $\varphi(x, y, t)$, i.e., $\bar{\varphi}(k, t)=\int_{-\infty}^{+\infty} \int_{-\infty}^{+\infty} \varphi(x, y, t)$ $\times \exp \left[-i\left(k_{x} x+k_{y} y\right)\right] d x d y$. In order to discretize Eq. (4) in the 
time domain for the use of the semi-implicit scheme, we deal with the terms in Eq. (4) separately. The key idea is that treating the linear terms implicitly can reduce the stability constraints, while treating the nonlinear term explicitly can avoid directly solving the nonlinear equation at each time step during the iteration. Consequently, we obtain the final form of the discretized equation,

$$
\begin{aligned}
\left(\bar{\varphi}^{n+1}-\bar{\varphi}^{n}\right) /(\Delta t)= & \left(k^{2} \bar{\varphi}^{n+1}-k^{2} \bar{\varphi}^{3} n-k^{4} \bar{\varphi}^{n+1}+k^{2} \bar{\beta}\right) \\
& -\alpha\left(\bar{\varphi}^{n+1}-\bar{\varphi}_{0}\right)
\end{aligned}
$$

which leads to the iteration format such that

$$
\bar{\varphi}^{n+1}=\frac{\bar{\varphi}^{n}+\left[\alpha \bar{\varphi}_{0}+k^{2}\left(\bar{\beta}-\overline{\varphi^{3}}\right)\right] \Delta t}{1+\left[\alpha+k^{2}\left(k^{2}-1\right)\right] \Delta t} .
$$

During the numerical iteration, we need to perform the double Fourier transform and its inverse at each time step that can be implemented by using the efficient twodimensional (2D) fast Fourier transform (2D-FFT) and its inverse (2D-IFFT). The typical operations from time step $n$ to $n+1$ are as follows:

(1) With $\varphi\left(x_{i}, y_{j}, t_{n}\right)$ and $\bar{\varphi}_{p q}\left(k, t_{n}\right)$ at time step $n$, evaluate $\varphi^{3}\left(x_{i}, y_{j}, t_{n}\right)$,

(2) perform 2D-FFT to determine $\overline{\varphi_{p q}^{3}}\left(k, t_{n}\right)$,

(3) evaluate relation (6) to determine $\bar{\varphi}_{p q}\left(k, t_{n+1}\right)$, and

(4) perform 2D-IFFT to invert $\bar{\varphi}_{p q}\left(k, t_{n+1}\right)$ and then obtain $\varphi\left(x_{i}, y_{j}, t_{n+1}\right)$ at time step $n+1$.

The main computations above can be efficiently implemented by means of both 2D-FFT and 2D-IFFT, and the time steps can be selected as orders of the conventional ones used in explicit Euler forward method because of the use of semiimplicit algorithm in the time integration. It can be shown and has been verified by numerical experiments ${ }^{46,47}$ that the semi-implicit Fourier-spectral method is unconditionally stable in the sense that for a fixed set of physical parameters there always exists a time threshold $\Delta t_{c}$, independent of the spatial grid size, such that for all $\Delta t \leqslant \Delta t_{c}$ the above scheme is stable for all spatial grid sizes. As a result, the semiimplicit Fourier-spectral method can significantly enhance the computational efficiency and improve the numerical stability and therefore is more efficient than the regular finite difference methods used for cell dynamics simulation of phase separation in BCPs as demonstrated in the present study as follows.

\section{NUMERICAL RESULTS}

Within the framework formulated in Sec. II, now let us consider the phase-segregation process of the BCP films on a flat homogeneous substrate and on a flat, chemically pattered substrate, respectively. The control parameters of the $\mathrm{BCP}$ films used in this study include the factors $\alpha$ and $\varphi_{0}$ and the $\beta$ matrix. We investigate the effects of these parameters on the phase-segregation process by using a $2 \mathrm{D}$ computational cell under periodic boundary conditions. The time step used for the all time-integrations in this study is fixed as 0.5 , and the initial concentration of the order parameter $\varphi$ is assumed to have a Gaussian fluctuation of magnitude of 0.002 around the average values designated in the following.

\section{A. BCP films on homogeneous substrates}

In this case the $\beta$ matrix is a trivial constant matrix, therefore it does not influence the simulations according to Eq. (3). Hereafter, we first investigate the effects of the parameters $\alpha$ and $\varphi_{0}$ on the phase separation of a BCP film on a homogeneous substrate to show the efficiency of the present numerical scheme. In each simulation a 2D computational cell of size of $256 \times 256$ is used. We choose $\alpha=0.2$ and 0.1 for $\varphi_{0}=0$ (symmetric) to show the effect of BCP group incompatibility on the phase separation, and select $\alpha$ $=0.05$ and 0.02 for $\varphi_{0}=0.15$ and 0.35 (asymmetric) to show the effect of BCP asymmetry on the phase separation. After 5000 iterations, well-developed serpentine structures of the order parameter have formed in the symmetric BCP films, as shown in Figs. 1(a) and 1(b). Nevertheless, for greater $\alpha$ value the $A-B$ groups of the symmetric BCP films have not been totally separated after the given number of iterations due to the similar physiochemical groups under consideration.

For asymmetrical BCP films $\left(\varphi_{0} \neq 0\right)$, after 5000 iterations, simulation indicates that the phase separation leads to densely packed nanodots or nanoislands [see Figs. 1(c) and 1(d)]. The nanodots and nanoislands obtained under these conditions are randomly distributed and therefore have limited values in view of practical applications, especially in microelectronics. Therefore, it is desirable to further consider the process that guides the phase separation of these BCP films through a chemically patterned substrate as mentioned in Sec. I.

\section{B. BCP films on chemically patterned substrates}

In this case the nontrivial $\beta$ matrix is adopted to model the heterogeneity of the surface chemical potential. An artificial pattern of alternating parallel strips and spaces with equal width, as illustrated in Fig. 2(a), has been considered in recent experimental and simulation studies. ${ }^{15,18,20} \mathrm{~A}$ chemically mosaic-patterned substrate, as shown in Fig. 2(c), has also been examined in recent lattice Monte Carlo simulations. ${ }^{41-43}$ These studies provided valuable information in understanding the guided phase-separation process of BCP films and for comparison. Hereafter, we consider the surface chemical potentials characterized through the pattern semiperiod $w$ and the potential magnitude $U$ similar to those used in the literature. ${ }^{33}$ We set a positive constant $U$ at the $A$-preferring surface potential domain while assuming zero potential at the domain of neutral surface, as illustrated in Fig. 2. Without loss of generality, we fix $\alpha=0.1$ and $U$ $=0.001$ for the phase-separation simulation of the symmetric BCP films and $\alpha=0.02, \varphi_{0}=0.35$, and $U=0.001$ for the asymmetric ones. We aim to examine the effect of $w$ on the film morphology development, since this effect is vital to nanomanufacturing. ${ }^{15,18,20}$

For symmetric BCP films with $\alpha=0.1$, numerical simulation determined the unconstrained lamella period as $\lambda^{*}$ $\approx 10$ which can also be estimated analytically. ${ }^{24,33}$ In order to 


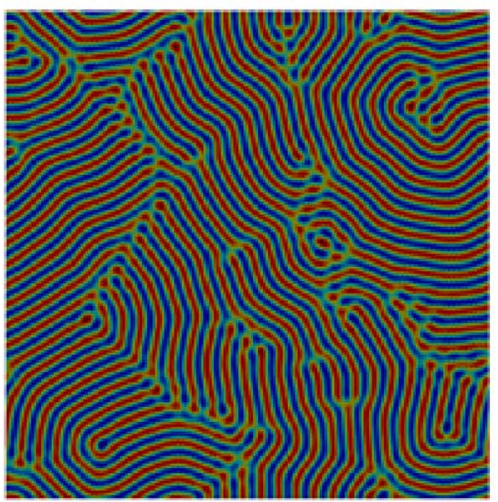

(a)

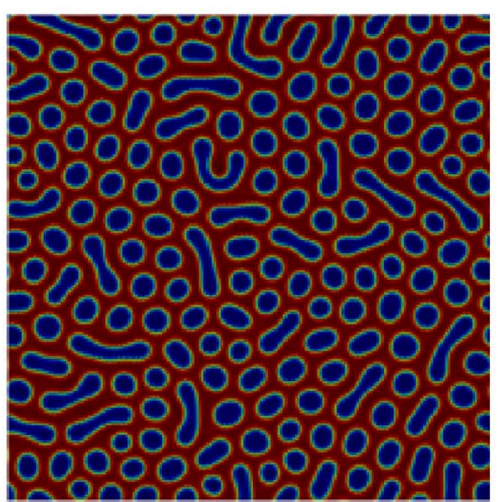

(c)
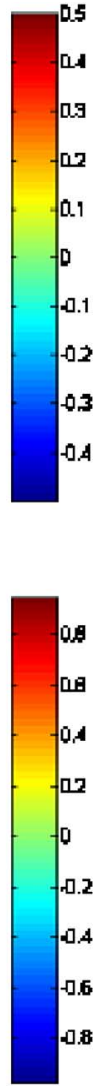

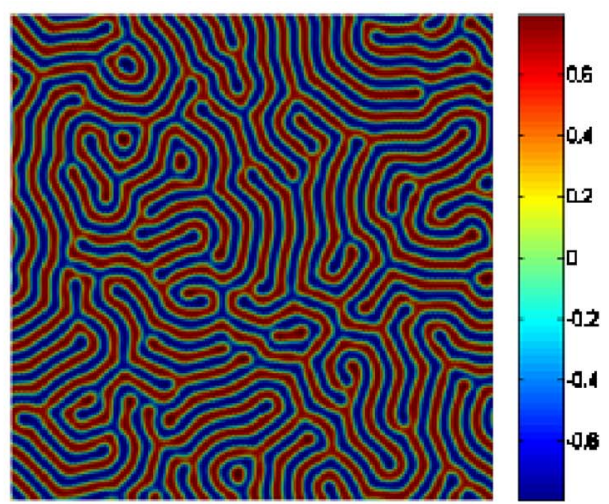

(b)

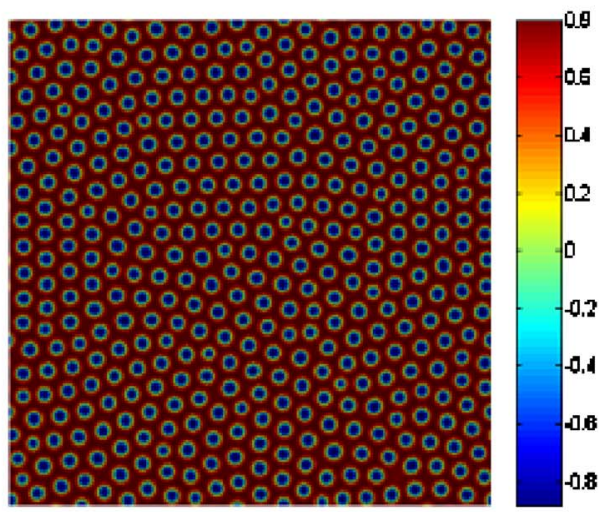

(d)
FIG. 1. Morphology of the order parameter $\varphi$ of BCP films after 5000 iterations. (a) $\alpha=0.2$ and $\varphi_{0}=0$ (symmetric), (b) $\quad \alpha=0.1$ and $\varphi_{0}=0$ (symmetric), (c) $\alpha=0.02$ and $\varphi_{0}$ $=0.15$ (asymmetric), and (d) $\alpha=0.05$ and $\varphi_{0}=0.35$ (asymmetric). satisfy the exact periodic boundary conditions, we employ the computational cell with varying size. After 5000 iterations, the order morphologies have evolved very well (see Fig. 3). When the pattern period $(2 w=8)$ is slightly below the unconstrained lamellar period $\lambda^{*}$ [see Fig. 3(a)], due to the localized attraction induced by the incompatible heterogeneous substrate, the formed lamella pattern is compressed, and dislocations exist. However, most of the formed pattern is registered and epitaxial to the surface pattern, same as those observed in recent experiments. ${ }^{15}$ When the pattern period $(2 w=10)$ is very close to $\lambda^{*}$, highly orientated lamellae are formed through guided self-assembly [see Fig. 3(b)]. due to its specific pattern period corresponding to $\lambda^{*}$. Furthermore, when the pattern period $(2 w=12)$ is slightly greater than $\lambda^{*}$, as shown in Fig. 3(c), wavy lamellae pattern

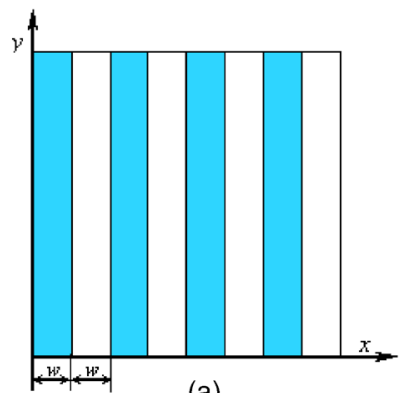

(a)

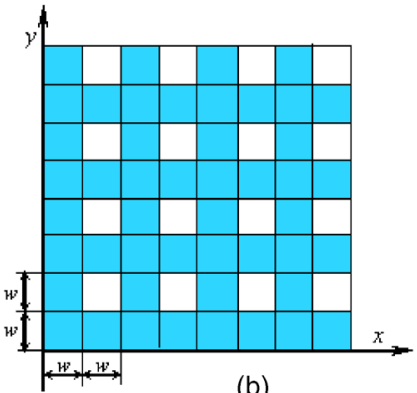

(b)
A-preferring surface notential domain We call this pattern as standing pattern or resonant pattern

FIG. 2. Schematic of two chemically patterned substrates. (a) Striped pattern and (b) mosaic pattern. is formed. This agrees with the recent experimental observation $^{15}$ and is also very close to the order profile given by Chen and Chakrabarti. ${ }^{33}$ Consequently, when the pattern period $(2 w=14)$ is much greater than $\lambda^{*}$ [see Fig. 3(d)], welldeveloped serpentine structures are formed. Clearly, patterned surface chemical potential in this case has no significant effect on the phase separation. This is also confirmed in recent experiments. ${ }^{15}$

Clearly, the observed surface morphology of the symmetric BCP films is strongly influenced by the commensurability between the unconstrained natural period $\lambda^{*}$ and the pattern period. In the absence of any surface confinement and surface chemical potential, the lamella formation is largely a This is made according to the balance between the interfacial energy due to the formation $A-B$ interface and the nonlocal molecular repulsion. As a result, it leads to the serpentine structure of period $\lambda^{*}$ based on the original locations of the $A$ and $B$ monomers in the BCP film and the extent of initial thermal fluctuation, as shown in Figs. 1(a) and 1(b). For a chemically patterned surface of alternating parallel strips of $A$-preferring strips and neutral spaces, when the pattern period is very close to $\lambda^{*}$, the $A$ and $B$ monomers can mostly accommodate the pattern to form the parallel lamellae. Though the initial locations of $A$ and $B$ monomers are randomly distributed, after a quench, the $A$ monomers diffuse to the locations of the $A$-preferring strips in order to reduce the system energy. During this process, the $A$ monomers initially staying on the $A$-preferring strips keep the same location and may also rotate to accommodate the motion of the $B$ monostochastic process reducing the free energy of the BCP film. 


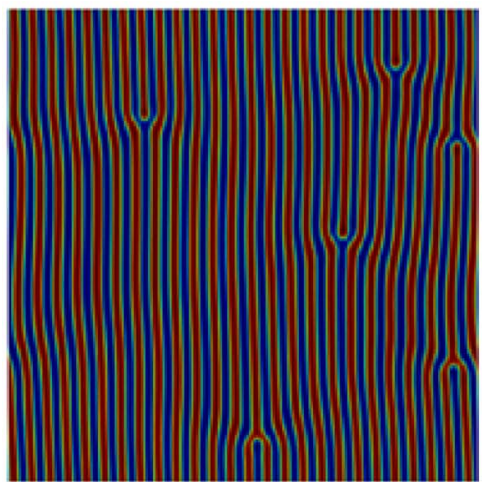

(a)

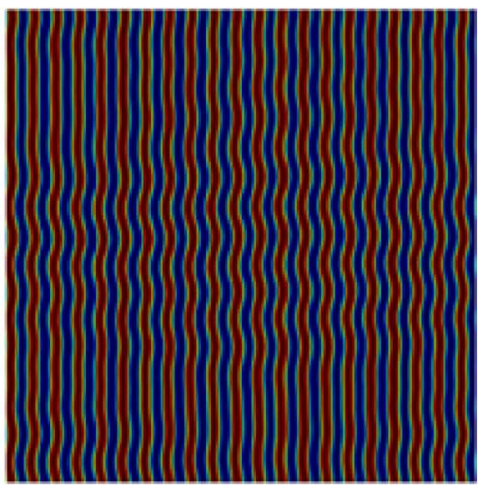

(c)
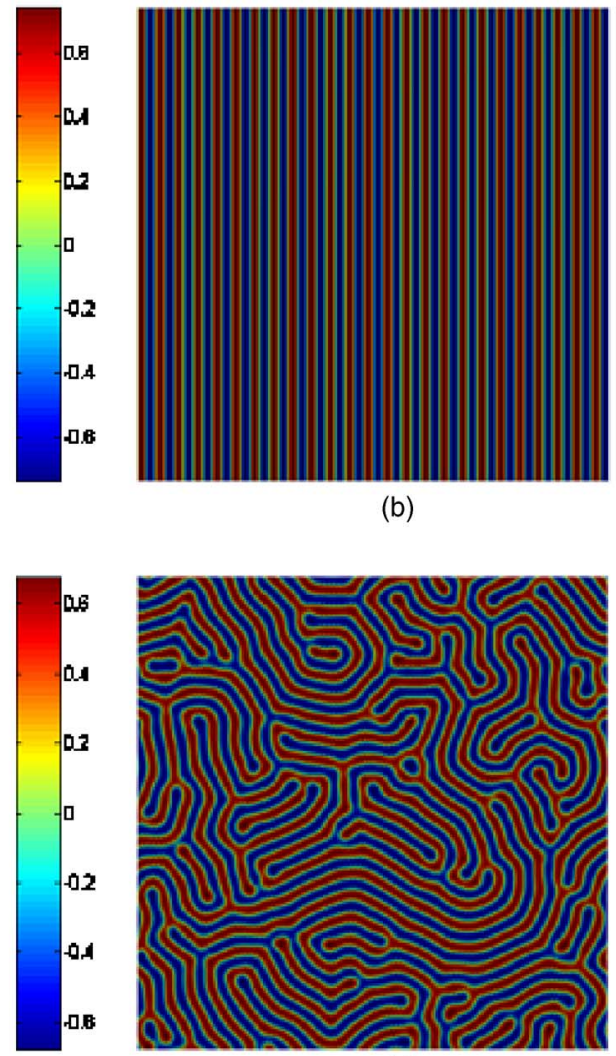

(d)

(b)
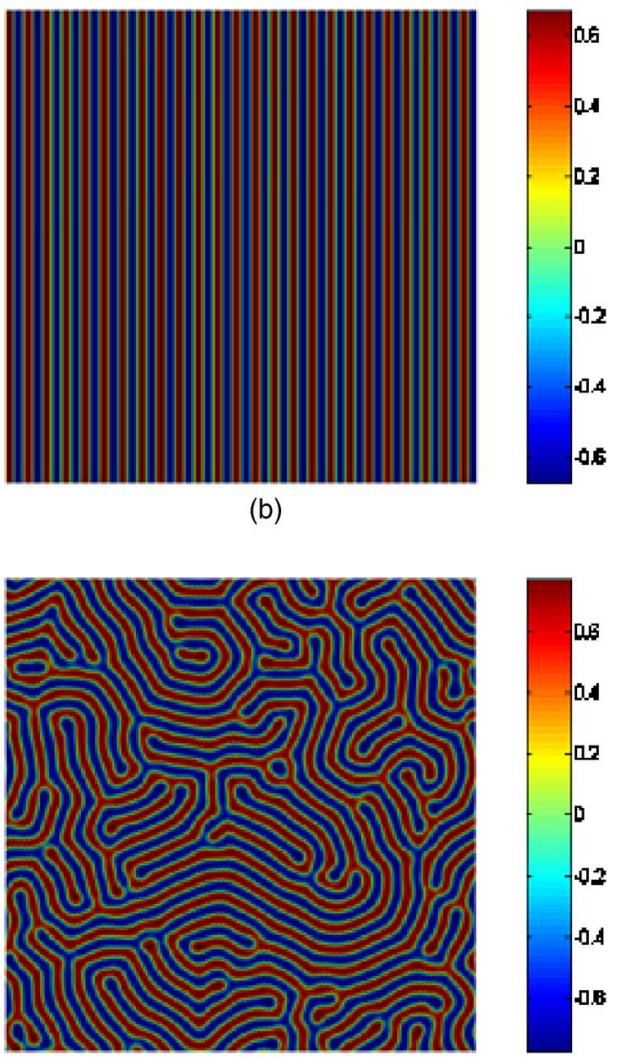

FIG. 3. Morphology of the order parameter $\varphi$ of symmetric BCP films on chemically patterned substrates after 5000 iterations. (a) $w=4$ (cell size: $256 \times 256$ ), (b) $w=5$ (cell size: 250 $\times 250$ ), (c) $w=6$ (cell size: $252 \times 252$ ), and (d) $w=7$ (cell size: $252 \times 252$ ). mers; meanwhile the $A$ monomers initially staying on the neutral strips will diffuse to the neighboring $A$-preferring strips by exchanging the locations of the $B$ monomers that stay on the $A$-preferring strips. Due to this specific pattern period $\lambda^{*}$, all $A$ monomers can be totally registered along the $A$-preferring strips, and the left $B$ monomers are totally registered along the neutral spaces. After sufficient time, the microstructure of the BCP film will finally evolve into the highly ordered $[-A-B-A-B-A-B-]$ format, as observed in experiments. ${ }^{15,18,20}$ However, when the pattern period is below and above $\lambda^{*}$, after a quench, the $A$ monomers try to register along the $A$-preferring strips. This registration is constrained by the $B$ monomers due to the unique internal chain structure of the BCP film. In the case of pattern period below $\lambda^{*}$ though, most $A$ monomers are registered along the parallel $A$-preferring strips. Due to the mismatch between the unconstrained lamella period $\lambda^{*}$ and the artificial pattern period, the formed lamellae are compressed and therefore are not fully aligned [see Fig. 3(a)]. Because of the chain length constraint of the $A-B \mathrm{BCP}$ film, the compression leads to dislocation, i.e., the breakage of the lamellae at some locations. In the case of pattern period slightly above $\lambda^{*}$, the lamellar ordering in the BCP film always tries to accommodate the surface pattern length scale by switching the lamellae with respect to the $A$-preferring strips according to an angle [see Fig. 3(c)]. This favors the evolution mechanism as discussed by Petera and Muthukumar. ${ }^{27,28}$ However, in the case of pattern period well above $\lambda^{*}$, because of the large portion of the homogeneous surface $(A$-preferring strips or neutral spaces) and the mismatch of the length scale, signifi- cant $A$ monomers cannot diffuse to follow the pattern, therefore only slight lamella orientation can be detected [see Fig. 3(d)].

As a result, the present simulation has indicated that chemically patterned surface potential with period close to $\lambda^{*}$ leads to highly ordered surface morphology for symmetric BCP films, and it also significantly accelerates the guided self-assembly process. Using the above numerical scheme, we further investigate the phase-separation process of symmetric BCP films confined on a substrate surface with two different mosaic patterns of surface chemical potential [see Figs. 2(b) and 5(a)]. Simulation shows that the phase separation of the symmetric $\mathrm{BCP}$ film can hardly be guided to follow either mosaic pattern with any proper period, though recent lattice MC simulation showed some kind of surface ordering on mosaic surface for the recognition of surface chemical potential. $^{41-43}$

Furthermore, for an asymmetric BCP system with $\alpha$ $=0.02$ and $\varphi_{0}=0.35$, simulation determines the unconstrained natural period as $\lambda^{*} \approx 16$. When the magnitude of the chemical potential at $A$-preferring strips is selected as $U=0.001$, all the simulations are performed in the computational cell of size $256 \times 256$. Well-developed surface morphologies after 5000 iterations are shown in Fig. 4, from which it can be clearly observed that the films evolve into densely packed nanodots. When the pattern period $(2 w=8$ or 32$)$ is either much lower or much greater than $\lambda^{*}$, the resulting nanodots in Figs. 4(a) and 4(b) have weak orientation to follow the surface pattern. However, when the pattern period $(2 w=16)$ is very close to $\lambda^{*}$, the resulting nanodots are highly orientated, following the parallel $A$-preferring strips [see Fig. 


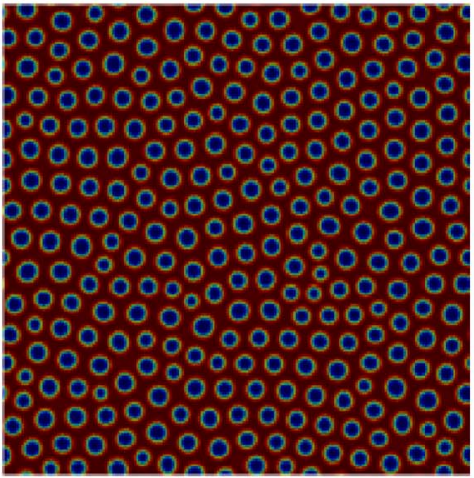

(a)

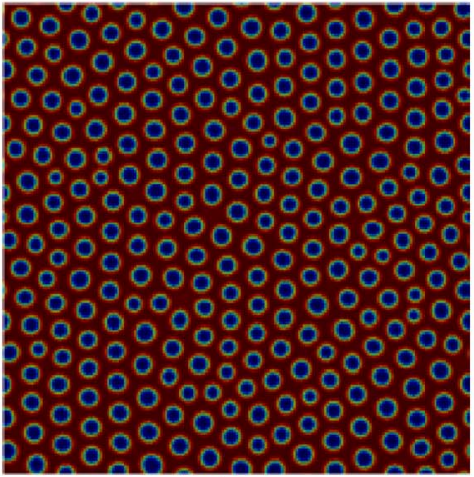

(c)
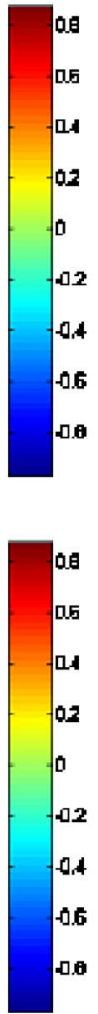

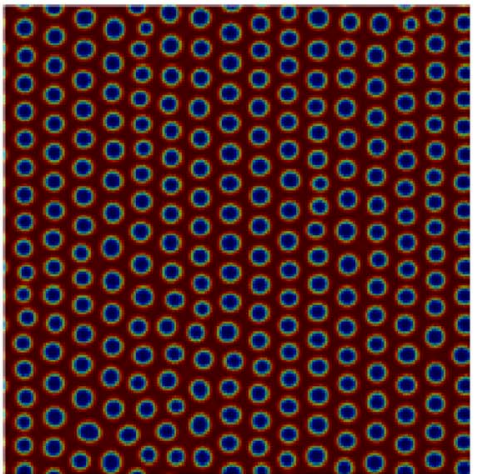

(b)

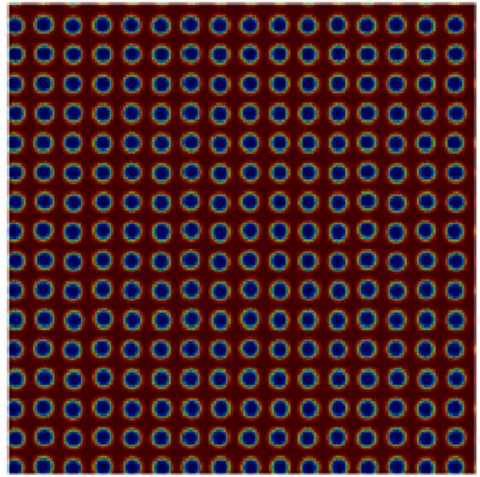

(d)
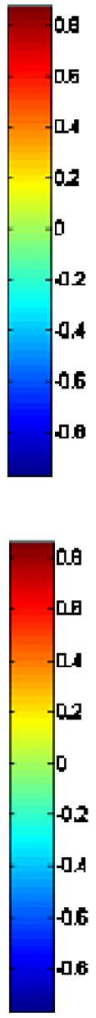

FIG. 4. Morphology of the order parameter $\varphi$ of asymmetric BCP films on chemically patterned substrates after 5000 iterations $(\alpha=0.02$ and $\varphi_{0}=0.35$ ). (a) $w=4$ (striped pattern), (b) $w=8$ (striped pattern), (c) $w=16$ (striped pattern), and (d) $w=8$ (mosaic pattern). 4(b)]. By comparison with the lamellae formed in symmetric BCP films, the nanodots formed in asymmetric BCPs are more oriented on the same patterned surfaces. The main reason is that for asymmetric $\mathrm{BCP}$ films $A$ and $B$ monomers have higher motion freedom due to their special asymmetric microstructure. For mosaic pattern [Fig. 2(b)] with period $(2 w=16)$, the surface morphology of the BCP film is highly ordered in both horizontal and vertical directions as expected. This mosaic pattern can be employed to produce highly aligned densely packed nanodots for microelectronics applications.

From Fig. 4 and other similar simulations performed in this study, we find that chemically patterned surface with period close to the unconstrained natural period $\lambda^{*}$ of the BCP films can dramatically accelerate the phase-separation process. We denominate this phenomenon as resonant phase separation, very similar to resonant vibration in physics. Therefore, under proper circumstances, periodic external fields with proper wavelength can be employed to accelerate the guided phase-segregation process of the BCP films. These external fields can be surface chemical potential, light radiation, thermal gradient, and electromagnetic fields, among others.

As an example, we also use the mosaic pattern $(2 w$ $=16$ and $U=0.001$ ) as shown in Fig. 5(a) to simulate the phase-separation process in the same asymmetric BCP film ( $\alpha=0.02$ and $\varphi_{0}=0.35$ ). Figure 5(b) shows the corresponding order parameter morphology after 5000 iterations. Clearly, in view of forming highly ordered morphology of BCP films, the pattern shown in Fig. 2(b) is better than the one in Fig. 5(a).

\section{Domain size evolution}

Above, we have investigated the morphology of BCP films after a sufficient number of iterations through phase-

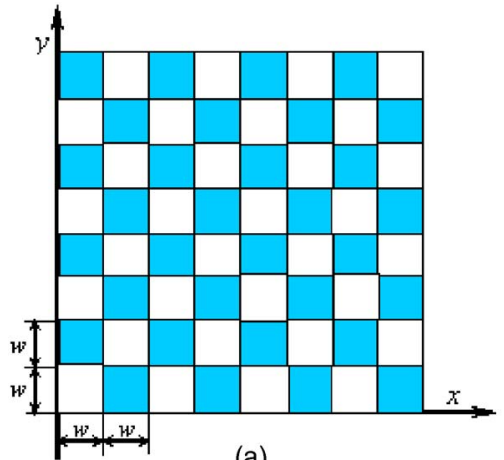

(a)

A-torefer ring surface notential dom ain

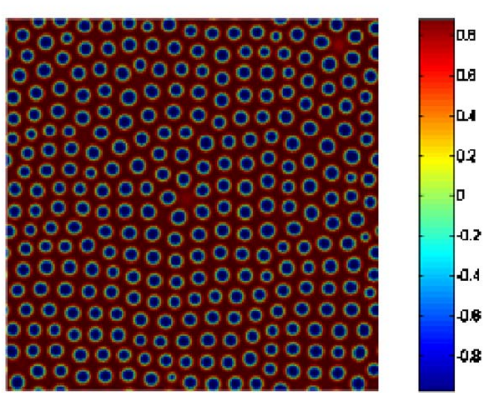

(b)

Weutral surface potential domain
FIG. 5. Pattern of surface chemical potential and resulting morphology of the order parameter $\varphi$ of an asymmetric BCP film $\left(\alpha=0.02\right.$ and $\left.\varphi_{0}=0.35\right)$. (a) Mosaicpatterned surface chemical potential $(w=8)$ and (b) resulting morphology of the order parameter $\varphi$ after 5000 iterations. 


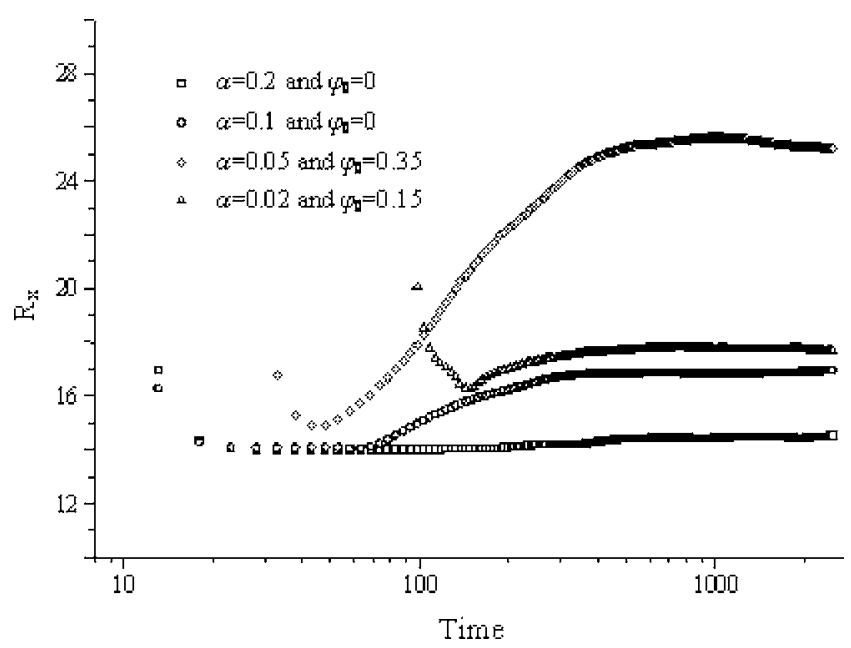

FIG. 6. Variation of the domain size $R_{x}(t)$ vs the time of four symmetric BCP systems.

separation modeling. In order to characterize the evolution of domain size, here we introduce the transient average domain size $R(t)$ along $x$ and $y$ directions that can be derived from the inverse of the transient average wave numbers $\left\langle k_{x}(t)\right\rangle$ and $\left\langle k_{y}(t)\right\rangle$ such that ${ }^{49-55}$

$$
R_{x}(t)=2 \pi /\left\langle k_{x}(t)\right\rangle, \quad R_{y}(t)=2 \pi /\left\langle k_{y}(t)\right\rangle,
$$

where

$$
\begin{aligned}
\left\langle k_{x}(t)\right\rangle & =\frac{\sum_{k_{x}} \sum_{k_{y}} k_{x} S\left(k_{x}, k_{y}, t\right)}{\sum_{k_{x}} \sum_{k_{y}} S\left(k_{x}, k_{y}, t\right)}, \\
\left\langle k_{y}(t)\right\rangle & =\frac{\sum_{k_{x}} \Sigma_{k_{y}} k_{y} S\left(k_{x}, k_{y}, t\right)}{\sum_{k_{x}} \sum_{k_{y}} S\left(k_{x}, k_{y}, t\right)} .
\end{aligned}
$$

In relations (7) and (8) $k_{x}$ and $k_{y}$ are the variables in Fourier domain and $S\left(k_{x}, k_{y}, t\right)$ is the element of the structure factor matrix defined by ${ }^{49-55}$

$$
S\left(k_{x}, k_{y}, t\right)=\frac{1}{N} \bar{\varphi}\left(k_{x}, k_{y}, t\right) \bar{\varphi}\left(-k_{x},-k_{y}, t\right)-\langle\varphi\rangle^{2} I\left(k_{x}, k_{y}\right),
$$

where $N=L^{2}$ is the total number of grids within the computational cell, $\langle\varphi\rangle$ is the average order parameter, and $\bar{\varphi}\left(k_{x}, k_{y}, t\right)$ and $I\left(k_{x}, k_{y}\right)$ are the coefficients of the 2D-FFT of $\varphi(x, y, t)$ and the unit in the computational cell, respectively. With the aid of definition (7), we consider the evolution of the domain size of the BCP systems in the above simulations as shown in Figs. 6-9.

Figures 6 and 7 show the variation of the domain size along $x$ and $y$ directions versus the time of four types of symmetric BCP films on homogeneous substrates as also shown in Fig. 1. In these cases, the domains of both symmetric and asymmetric BCP films evolve in an approximately isotropic way, i.e., each $R_{x}(t)$ curve is very close to the corresponding $R_{y}(t)$ curve. The final domain size can be identified from Figs. 6 and 7. For symmetric BCP films, the domain size is close to 14 for the BCP system of $\alpha=0.2$ and 17 for that of $\alpha=0.1$; for asymmetric BCP films, the domain size is close to 26 for the BCP system of $\alpha=0.05$ and $\varphi_{0}$ $=0.35$ and 18 for that of $\alpha=0.02$ and $\varphi_{0}=0.15$. The $R_{x}(t)$ and

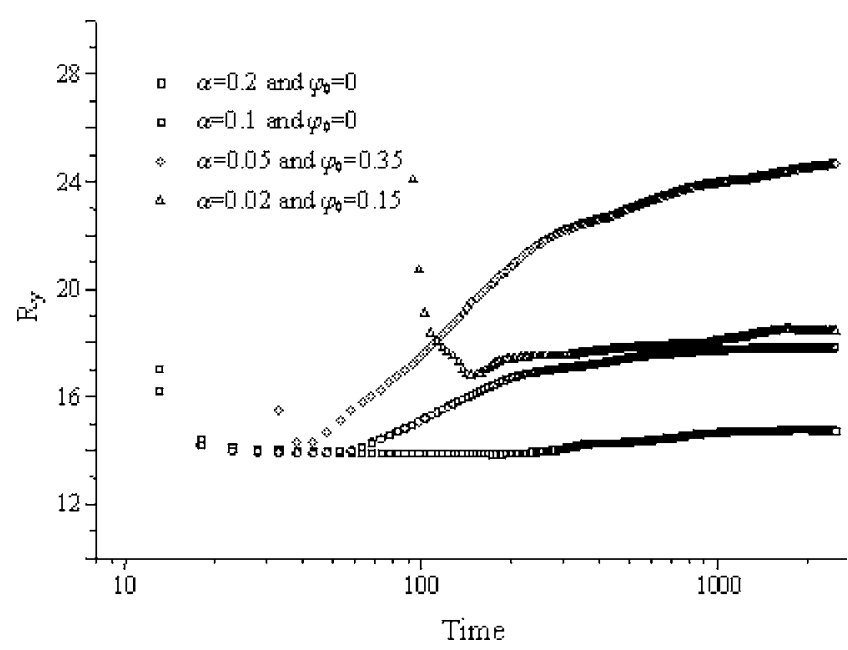

FIG. 7. Variation of the domain size $R_{y}(t)$ vs the time of four symmetric BCP systems.

$R_{y}(t)$ curves in Figs. 6 and 7 also indicate the growth rates of the domain size. It can be found that the domain size of the symmetric BCP system with $\alpha=0.1$ is almost constant during the entire phase-separation process. This implies that once the serpentine structures appear through initial nucleation, they maintain almost the same structures afterwards. This has been confirmed by the morphology evolution in the above simulation. However, for the asymmetric BCP system with $\alpha=0.05$ and $\varphi_{0}=0.15$, the domain size has the highest growth rate. During this process, very fine nanodots first appear through initial nucleation, and then these nanodots further coarsen, grow, and finally coalesce through diffusion the final equilibrium state. Accordingly, the domain size grows rapidly to the final size.

Figure 8 shows the variation of the domain size $R_{x}(t)$ versus the time along $x$ direction for the symmetric BCP film with $\alpha=0.1$ on chemically patterned surfaces as shown in Figs. 2(a) and 3. For this BCP system, the unconstrained natural period $\lambda^{*}$ is close to 10 . When the pattern period is $2 w=8$ or $2 w=10$ the final domain size is very close to $\lambda^{*}$

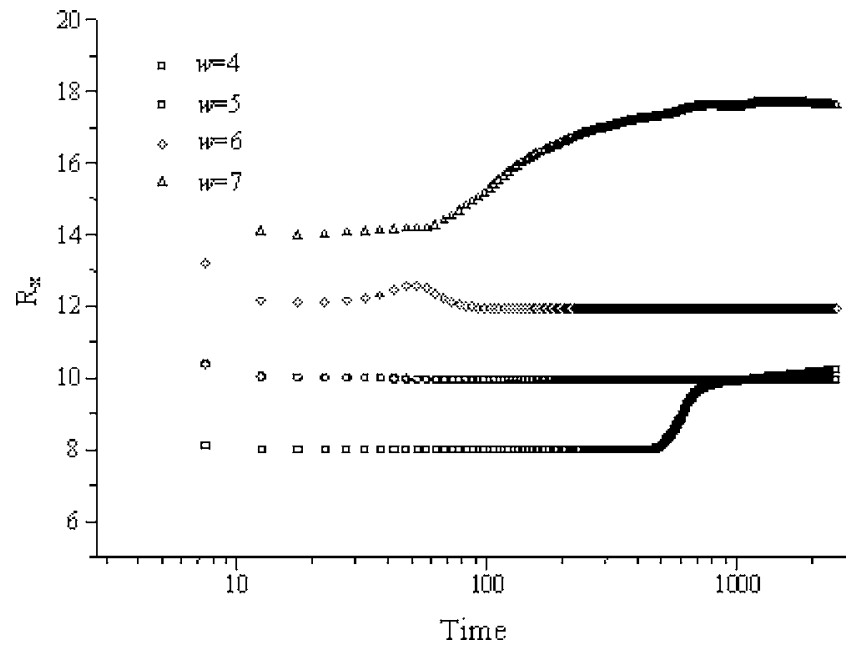

FIG. 8. Variation of the domain size $R_{x}(t)$ vs the time of a symmetric BCP film $\left(\alpha=0.1\right.$ and $\left.\varphi_{0}=0.0\right)$ on a chemically patterned substrate with four pattern wavelengths of $2 w=8,10,12$, and 14 , respectively. 


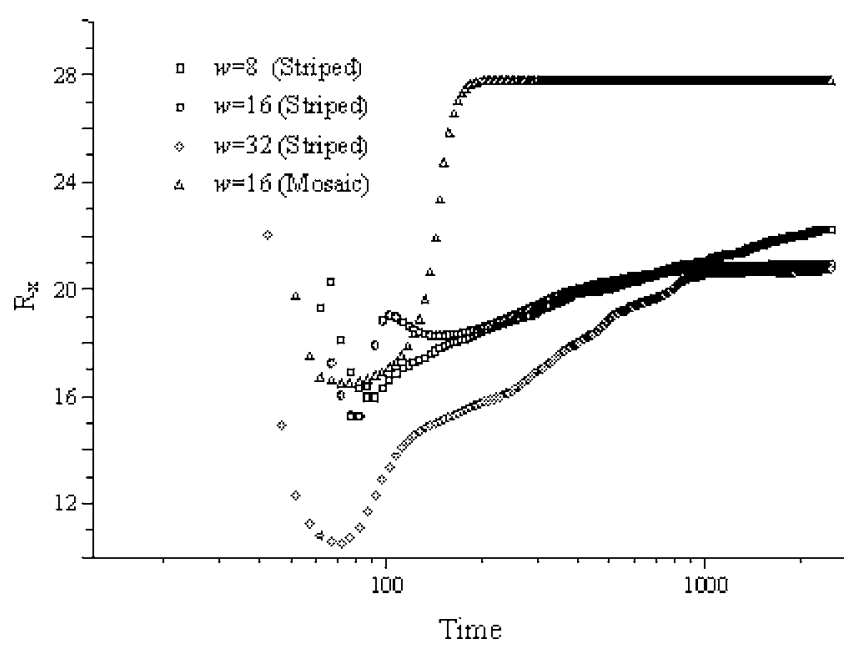

FIG. 9. Variation of the domain size $R_{x}(t)$ vs the time of an asymmetric BCP film $\left(\alpha=0.02\right.$ and $\left.\varphi_{0}=0.35\right)$ on a chemically patterned substrate with four pattern wavelengths of $2 w=8,16,32$, and 16 (mosaic), respectively.

$\approx 10$ in Fig. 8 . Therefore, these two pattern periods can be employed to produce BCP films with highly orientated microstructures as shown in Figs. 3(a) and 3(b). Furthermore, when the pattern period is slightly greater than $\lambda^{*} \approx 10$, e.g., $2 w=12$ in this study, the final domain size is modulated by the patterned surface as 12, shown in Fig. 3(c). Consequently, for pattern period $2 w=14$ or greater, the domain grows almost isotropically, i.e., patterned surface chemical potential does not influence the phase separation as shown in Fig. 3(d). The final domain size in this case is close to 17 from Fig. 8.

Figures 9 and 10 show the variation of the domain size along $x$ and $y$ directions versus the time of the asymmetric $\mathrm{BCP}$ on a chemically patterned substrate with four patterns, respectively (see Figs. 2 and 4). After 5000 iterations, as shown in Fig. 9, the domain size is still growing in the cases of the pattern periods with 8 and 32. However, the domain has developed well in the case of pattern period 16. This is also confirmed in Fig. 4, where growing nanodots can be clearly detected, as shown in Figs. 4(a) and 4(c). Thus, pat-

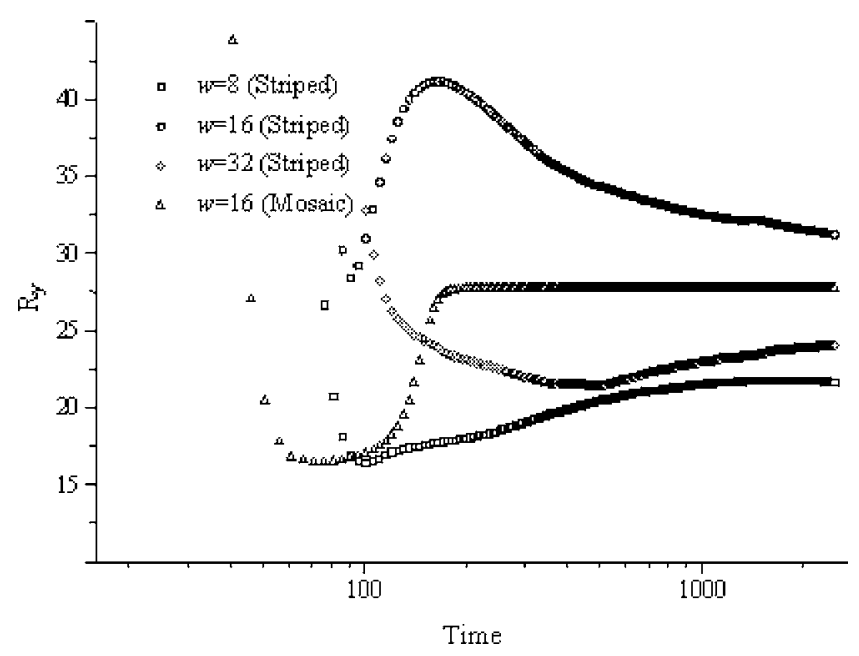

FIG. 10. Variation of the domain size $R_{y}(t)$ vs the time of an asymmetric BCP film $\left(\alpha=0.02\right.$ and $\left.\varphi_{0}=0.35\right)$ on a chemically patterned substrate with four pattern wavelengths of $2 w=8,16,32$, and 16 (mosaic), respectively. tern period has significant effect on the domain growth of $\mathrm{BCP}$ films. When the pattern period is close to the unconstrained natural period of the $\mathrm{BCP}$ films, patterned substrate can be utilized to enhance the phase-separation rate.

Furthermore, from Figs. 9 and 10, it can also be found that the final domain size based on definition (7) is slightly greater than the one detected by naked eyes in Fig. 4, especially in the case of mosaic patterns. In order to explain this deviation, we compare the order parameters at different grids and simultaneously check the structure matrix $S\left(k_{x}, k_{y}, t\right)$ after a sufficient number of iterations. It is found that there always exists a small deviation of the order parameters at grids located at the periodic positions, and the structure matrix also maintains very small nonzero values at the elements of the nondominate wave numbers. This deviation cannot be totally eliminated through increasing the number of iterations. In the sense of physics, due to the existence of the patterned surface, there exists an energy barrier between two neighboring locations (nanodots), and the phase boundary energy of nanodots also contributes part of this energy barrier. Therefore, after sufficient iterations, a small quantity of deviation of the order parameters at neighboring isolated nanodots cannot be offset through particle (molecular) diffusion that should overcome this energy barrier. This is also the limitation of definition (7) in determining the domain size. As a matter of fact, the computed domain size based on the mosaic patterns is greater than that found in Fig. 4(d). However, the peak $k_{x}$ and $k_{y}$ values in the structure factor matrix exactly accord to the domain sizes as observed in the morphology patterns. Thus, a proper window function may be adopted to filter the numerical noises and therefore to properly indicate the actual visual domain size. For the surface pattern of alternating parallel strips of chemical potential, Fig. 3 does not show this deviation for the symmetric $\mathrm{BCP}$ films, since in this case the $\mathrm{BCP} A$ monomers can diffuse freely along the $A$-preferring strips in the vertical direction.

\section{SUMMARY AND CONCLUSIONS}

In this study we have examined the phase-segregation process of symmetric/asymmetric BCP films on heterogeneous substrates with patterned chemical potential by solving the resulting $\mathrm{CH}$ equation based on an efficient semi-implicit Fourier-spectral algorithm. We have evaluated the effects of pattern scale, surface chemical potential, and BCP asymmetry on the self-assembly process of the BCP films. We find that the morphology of both symmetric and asymmetric $\mathrm{BCP}$ films is strongly influenced by the commensurability between the unconstrained natural period $\lambda^{*}$ and the artificial pattern period. For patterned surface with alternating parallel strips of chemical potential, when the pattern period is close to $\lambda^{*}$, the symmetric BCP films form highly ordered lamella structures of period $\lambda^{*}$ and the asymmetric BCP films form highly oriental nanodots on parallel lines of period $\lambda^{*}$. When the pattern period is lower than $\lambda^{*}$, the symmetric BCP films form compressed lamella structures following the pattern and dislocations exist, and the asymmetric BCP films form disordered nanodots with weak orientation. When the pattern period is slightly greater than $\lambda^{*}$, the symmetric BCP films 
form wavy lamella structures following the pattern. When the pattern period is well above $\lambda^{*}$, patterned surface has no significant effect on the phase separation of both symmetric and asymmetric BCP films. Chemically patterned surface with period close to $\lambda^{*}$ is capable of dramatically accelerating the guided self-assembly process. Present numerical results of the symmetric BCP films agree very well with the recent experimental results, and the simulation results of the asymmetric BCP films expect a potential nanomanufacturing method to produce highly ordered nanodots in two directions through the guided self-assembly of asymmetric BCP films on chemically patterned substrates.

Nevertheless, for a realistic BCP system, in order to implement the coarse-grained phase-separation modeling as discussed in this work, one needs to know the exact free energy of the BCP system by experimental investigation or detailed $a b$ initio calculation. To this end, if the system is given, the guided self-assembly of the BCP films can be predicted exactly using the present efficient method. Moreover, for BCPs with sufficient thickness or in solutions, the guided self-assembly process should also take into account the 3D effect and multiple phases that will be discussed somewhere else.

\section{ACKNOWLEDGMENT}

Partial support of this work by the U.S. ARO/ARL and the U.S. AFOSR is greatly appreciated.

${ }^{1}$ I. W. Hamley, The Physics of Block Copolymer (Oxford University Press, Oxford, 1998).

${ }^{2}$ N. Hadjichristidis, S. Pispas, and G. Floudas, Block Copolymer-Synthetic Strategies, Physical Properties, and Application (Wiley, New York, 2003).

${ }^{3}$ V. Castelletto and I. W. Hamley, Curr. Opin. Solid State Mater. Sci. 8, 426 (2004).

${ }^{4}$ M. J. Fasolka and A. M. Mayes, Annu. Rev. Mater. Res. 31, 323 (2001).

${ }^{5}$ M. V. Tirrell and A. Katz, MRS Bull. 30, 700 (2005).

${ }^{6}$ M. D. Ward, MRS Bull. 30, 705 (2005).

${ }^{7}$ T. Bein, MRS Bull. 30, 713 (2005).

${ }^{8}$ J. Yoon, W. Lee, and E. L. Thomas, MRS Bull. 30, 721 (2005).

${ }^{9}$ H. Colfen and S.-H. Yu, MRS Bull. 30, 727 (2005).

${ }^{10}$ M. Boncheva and G. M. Whitesides, MRS Bull. 30, 736 (2005).

${ }^{11}$ C. J. Hawker and T. P. Russell, MRS Bull. 30, 952 (2005).

${ }^{12}$ M. Park, C. Harrison, P. M. Chaikin, R. A. Register, and D. H. Adamson, Science 276, 1401 (1997).

${ }^{13}$ L. Rockford, Y. Liu, P. Mansky, T. P. Russell, M. Yoon, and S. G. J. Mochrie, Phys. Rev. Lett. 82, 2602 (1999).

${ }^{14}$ W. A. Lopes and H. M. Jaeger, Nature (London) 414, 735 (2001).

${ }^{15}$ S. O. Kim, H. H. Solak, M. P. Stoykovich, N. J. Ferrier, J. J. de Pablo, and P. F. Nealey, Nature (London) 424, 411 (2003).

${ }^{16}$ J. Y. Cheng, A. M. Mayes, and C. A. Ross, Nat. Mater. 3, 823 (2004).

${ }^{17}$ N. Kumar and J. I. Hahm, Langmuir 21, 6652 (2005).

${ }^{18}$ M. P. Stoykovich, M. Muller, S. O. Kim, H. H. Solak, E. W. Edwards, J. J. de Pablo, and P. F. Nealey, Science 308, 1442 (2005).

${ }^{19}$ C. Harrison, D. H. Adamson, Z. Cheng, J. M. Sebastian, S. Sethuraman, D. A. Huse, R. A. Register, and P. M. Chaikin, Science 290, 1558 (2000).

${ }^{20}$ E. W. Edwards, M. P. Stoykovich, M. Muller, H. H. Solak, J. J. De Pablo, and P. F. Nealey, J. Polym. Sci., Part B: Polym. Phys. 43, 3444 (2005).

${ }^{21}$ I. Norde, Surface and Interfacial Aspects of Biomedical Applications (Plenum, New York, 1995).

${ }^{22}$ S. A. Brazovskii, Sov. Phys. JETP 41, 85 (1975).

${ }^{23}$ L. Leibler, Macromolecules 13, 1602 (1980).

${ }^{24}$ T. Ohta and K. Kawasaki, Macromolecules 19, 2621 (1986).

${ }^{25}$ G. H. Fredrickson, Macromolecules 20, 2535 (1987).

${ }^{26}$ G. H. Fredrickson and E. Helfand, J. Chem. Phys. 87, 697 (1987).

${ }^{27}$ D. Petera and M. Muthukumar, J. Chem. Phys. 107, 9640 (1997).

${ }^{28}$ D. Petera and M. Muthukumar, J. Chem. Phys. 109, 5101 (1998).

${ }^{29}$ Y. Tsori and D. Andelman, J. Chem. Phys. 115, 1970 (2001).

${ }^{30}$ Y. Tsori and D. Andelman, Europhys. Lett. 53, 722 (2001).

${ }^{31}$ Y. Tsori and D. Andelman, Macromolecules 34, 2719 (2001).

${ }^{32}$ G. Brown and A. Chakrabarti, J. Chem. Phys. 101, 3310 (1994).

${ }^{33}$ H. Chen and A. Chakrabarti, J. Chem. Phys. 108, 6897 (1998).

${ }^{34}$ I. Podariu and A. Chakrabarti, J. Chem. Phys. 113, 6423 (2000).

${ }^{35}$ Q. Wang, S. K. Nath, M. D. Graham, P. F. Nealey, and J. J. de Pablo, J. Chem. Phys. 112, 9996 (2000).

${ }^{36}$ S. Stepanow and A. A. Fedorenko, Europhys. Lett. 58, 368 (2002).

${ }^{37}$ Q. Wang, P. F. Nealey, and J. J. de Pablo, Macromolecules 36, 1731 (2003).

${ }^{38}$ I. Podariu and A. Chakrabarti, J. Chem. Phys. 118, 11249 (2003).

${ }^{39}$ D. P. Cao and J. Z. Wu, J. Chem. Phys. 122, 194703 (2005).

${ }^{40}$ X. H. He, M. Song, H. J. Liang, and C. Y. Pan, J. Chem. Phys. 114, 10510 (2001)

${ }^{41}$ J. J. Semler and J. Genzer, Macromol. Theory Simul. 13, 219 (2004).

${ }^{42}$ A. Jayaraman, C. K. Hall, and J. Genzer, Phys. Rev. Lett. 94, 078103 (2005).

${ }^{43}$ A. Jayaraman, C. K. Hall, and J. Genzer, J. Chem. Phys. 123, 124702 (2005).

${ }^{44}$ J. J. Christensen and A. J. Bray, Phys. Rev. E 58, 5364 (1998).

${ }^{45}$ P. Tang, F. Qiu, H. D. Zhang, and Y. L. Yang, Phys. Rev. E 72, 016710 (2005).

${ }^{46}$ L. Q. Chen and J. Shen, Comput. Phys. Commun. 108, 147 (1998).

${ }^{47}$ J. Z. Zhu, L. Q. Chen, J. Shen, and V. Tikare, Phys. Rev. E 60, 3564 (1999).

${ }^{48}$ P. C. Hohenberg and B. I. Halperin, Rev. Mod. Phys. 49, 435 (1977).

${ }^{49}$ Y. Oono and S. Puri, Phys. Rev. A 38, 434 (1988).

${ }^{50}$ S. Puri and Y. Oono, Phys. Rev. A 38, 1542 (1988).

${ }^{51}$ A. Shinozaki and Y. Oono, Phys. Rev. A 45, R2161 (1992).

${ }^{52}$ A. Chakrabarti, R. Toral, and J. D. Gunton, Phys. Rev. E 47, 3025 (1993).

${ }^{53}$ J.-J. Zhang, G. J. Jin, and Y. Q. Ma, Phys. Rev. E 71, 051803 (2005).

${ }^{54}$ J.-J. Zhang, G. Jin, and Y. Ma, Eur. Phys. J. E 18, 359 (2005).

${ }^{55}$ J.-J. Zhang, G. J. Jin, and Y. Q. Ma, J. Phys.: Condens. Matter 18, 835 (2006). 